\title{
Dissolution of iron oxides in the frozen solution
}

\author{
WONYONG ChOI, ${ }^{1}$ KitAe KIM, ${ }^{2}$ SUnil PAUl M. \\ MENACHERRY ${ }^{1}$ \\ ${ }^{1}$ Division of Environmental Science and Engineering, \\ Pohang University of Science and Technology \\ (POSTECH), Pohang 37673, Korea \\ ${ }^{2}$ Korea Polar Research Institute (KOPRI), Incheon 21990, \\ Korea
}

Ice is one of the most ubiquitous solids on Earth, being present in the atmosphere, terrestrial surface, and ocean environment. Many environmental reactions taking place in ice are significantly different compared to aqueous counterparts. We investigated some redox chemical reactions in ice, especially those which have environmental significance. First, we studied the reductive dissolution of iron oxides trapped in ice and investigated their relevance to bioavailability. The photoreductive dissolution of iron oxides proceeded slowly in aqueous solution but was significantly accelerated in polycrystalline ice with subsequently releasing more bioavailable ferrous iron upon thawing. The enhanced photogeneration of $\mathrm{Fe}(\mathrm{II})_{\mathrm{aq}}$ in ice was confirmed regardless of the type of iron oxides [hematite, maghemite, goethite] and the kind of electron donors. When iron oxides were trapped in ice, the release of ferric and ferrous ions upon thawing was significantly accelerated. The enhanced iron dissolution in ice was observed even under dark condition. The extent of dissolved iron was greatly affected by the kind of organic complexing ligands. The enhanced dissolution of naturally occurring metal oxides in ice can serve as an efficient pathway for supplying bioavailable metal ions to organisms in the environment. We also showed that the chemical reaction between iron oxides and iodide is markedly accelerated to produce bioavailable iron $\left(\mathrm{Fe}(\mathrm{II})_{\mathrm{aq}}\right)$ and triiodide $\left(\mathrm{I}_{3}\right.$ : evaporable in the form of $\left.\mathrm{I}_{2}\right)$ in frozen solution (both with and without light irradiation) while it is negligible in aqueous phase. This characteristic phenomenon in ice described above is mainly ascribed to the so called "freeze concentration effect". When water begins to solidify to ice, organic/inorganic solutes, protons, and dissolved gases are excluded from the ice crystals and subsequently concentrated in the liquid-like grain boundary region. The highly concentrated substrates in ice grain boundaries can cause distinct outcomes that are markedly different from the aqueous counterparts. The environmental redox chemical reactions occurring in ice may have significant effects on the chemical transformation processes in the frozen environment such as polar region, upper atmosphere, and frozen soil. 